\title{
Development and implementation of a mobile version of the O-SCORE assessment tool and case log for competency-based assessment in urology residency training: An initial assessment of utilization and acceptance amongst residents and faculty
}

\author{
Ryan Fitzpatrick, MD ${ }^{1}$; Nicholas R. Paterson, MD ${ }^{1}$; James Watterson, MD, FRCSC ${ }^{1}$; \\ Christine Seabrook, MEd ${ }^{2}$; Matthew Roberts, MD, MEd, FRCSC ${ }^{1}$ \\ ${ }^{1}$ Division of Urology, Department of Surgery; ${ }^{2}$ Office of Education, Department of Surgery; University of \\ Ottawa, Ottawa, ON, Canada
}

Cite as: Can Urol Assoc J 2018 July 31; Epub ahead of print. http://dx.doi.org/10.5489/cuaj.5482

Published online July 31, 2018

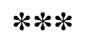

\section{Abstract}

Introduction: In competency-based models of residency training, work-based assessments of residents' technical skills are essential for both providing formative feedback and assessing surgical competence. The Ottawa Surgical Competency Operating Room Evaluation (O-SCORE) is a previously validated paper-based evaluation tool created to assess a surgical trainees' operative competence. To address some of the barriers to assessment, we developed and implemented a mobile application that combines the O-SCORE with a surgical case log.

Methods: A description of the development implementation process for the mobile OSCORE and case log is provided. Following implementation, a survey was developed and administered electronically to all faculty and residents within the University of Ottawa's Division of Urology to assess user perceptions and utilization of the application. The survey was administered and data collected via Survey Monkey.

Results: The overall response rate was $94 \%$. The majority of residents (94\%) reported that it was easy to log cases with the application, and $81 \%$ felt that it had a positive impact on their training; $75 \%$ of faculty were willing or very willing to complete evaluations when assigned and $66 \%$ felt that the application had a positive effect on the quality of feedback they provided.

Conclusions: Overall, faculty and residents felt that our mobile O-SCORE application was user-friendly and valuable as both a surgical log and assessment tool. With surgical programs moving towards competency-based models of training and assessment, the O- 
SCORE mobile application represents a practical electronic surgical log and work-based assessment instrument that can be easily adopted into any surgical training program.

\section{Introduction}

In surgical residency training, the assessment of residents' competence continues to be largely knowledge-based, with only a limited number of validated tools to assess and provide feedback for surgical and technical skills. ${ }^{1}$ While national certification bodies focus on written and oral examinations to determine competence, residency training programs are responsible for ensuring trainee's ability to perform all relevant operative procedures independently. Competence By Design (CBD) is an approach that focuses on learning the 'outcomes' or the abilities needed to practice, and involves designing training with an explicit progression of expertise, from novice to expert. Work-based assessments (WBAs) consist of observations in the workplace that provide performance rating information and feedback to the learner, and are shared with trainees in a way that guides learning improvement towards achieving competence. ${ }^{2}$ With the ongoing transition of residency programs nationwide to CBD, there is a need for validated WBAs. Traditionally, the assessment of surgical trainees' operative skills has been accomplished through informal intraoperative observations and feedback from faculty (which are rarely documented). In the absence of any formal documentation of a trainee's surgical experience, residency programs and some licensing bodies have relied on surgical case logs as surrogate measures of competence. However, surgical case logs alone lack content validity, as simply being in the operating room doesn't mean the trainee participated in the case or is competent to complete it independently. ${ }^{3}$

Assessment of resident performance during operative procedures can serve as an important feedback tool for trainees and to help trend performance. ${ }^{1}$ However, intraoperative assessments are administered at the discretion of individual institutions without standardization and their correlation with national board performance is limited and controversial. ${ }^{4}$ Surgical training programs should therefore have a reliable form of assessment to determine competency of a trainee by the attending physicians. An assessment tool that combines both operative evaluations and case logging on a userfriendly mobile platform was therefore developed to meet these needs.

The Ottawa Surgical Competency Operating Room Evaluation (O-SCORE) is a 9item surgical evaluation tool created to assess operative competence in surgical trainees. ${ }^{5}$ The development and validation of this tool are described elsewhere. ${ }^{5,6}$ The O-SCORE focuses on assessment of overall trainee competence to perform a specific procedure, and utilizes entrustment anchors such as "ability to safely perform this procedure independently" when comparing trainee performance to that of a fully qualified surgeon. These anchors align well with expert observer performance judgements. Ratings using 
both O-SCORE and the most validated technical skills assessment method (Objective Structured Assessment of Technical Skills) have demonstrated equivalence. ${ }^{7,8}$

The O-SCORE was initially validated in paper format, with the associated challenges of collecting and collating evaluation forms completed by multiple faculty across several training sites. The implementation and widespread use of iPads for clinical activities within The Ottawa Hospital provided an opportunity to transition the O-SCORE assessment to an electronic version. An electronic OSCORE application coupled with a surgical log was developed to provide a web-based application accessible on all mobile devices, tablets or desk-top computers. In this study, we describe the development and implementation process, and assess the utilization and user perceptions of the mobile OSCORE application within the University of Ottawa urology residency program.

\section{Methods}

Description of the electronic case log and O-SCORE application

The O-SCORE application is web-based and can be accessed on any mobile device. The case logging and assessment process begins when a resident logs into the application, selects the faculty they are working with from a drop-down list (Figure 1), and selects the case type from a drop-down menu of common procedures (Figure 2). The resident then saves the case and the date and time are logged.

For each logged procedure, a pre-set algorithm randomly selects operative cases to be evaluated. The frequency of assessment for each case type varies and is customizable by the program director; commonly-performed cases may be selected for evaluation on every third or fourth case that is logged, while less commonly performed cases may be selected every case that is logged. If a case is selected for evaluation, the staff physician immediately receives an e-mail notification. Ideally, cases are logged by the resident prior to beginning the procedure so that if the case is selected for evaluation the staff physician is notified prior to starting the operation. Once the case is complete, the staff physician then fills out the O-SCORE assessment form electronically (Figure 3). If the staff physician does not complete the online evaluation within 24 hours, the evaluation expires, which limits recall bias, thereby improving the reliability and validity of evaluations.

\section{Survey development}

A survey was developed to assess user perceptions and utilization of the O-SCORE application. The survey was administered electronically via Survey Monkey to all faculty and resident members of the University of Ottawa Division of Urology. Use of technology in the workplace, ease of use of the O-SCORE application and the use of the O-SCORE as a surgical log and evaluation tool were assessed. The respondents were not identifiable. 


\section{Results}

The overall response rate was 94\% (17/18 residents and 12/13 faculty). Twelve residents were in their senior years of training (PGY 3-5), and 5 were in their junior years (PGY 12). Five staff physicians were less than 40 years of age, 3 were between 40-49, 3 between 50-59 and one was older than 59.

\section{Resident responses}

Technology use in the workplace

Overall, responses indicate that residents were quite comfortable with using technology in the workplace. $100 \%$ of residents reported 1) that they were comfortable with using mobile technology like iPads and cell phones, and 2) that the O-SCORE application was easy to access on their devices. Prior to the launch of the O-SCORE application, 16/17 (94\%) of residents used the device on a daily basis for clinical work, and 15/17 (88\%) felt the wireless internet connectivity through the hospital networks was reliable. 14/17 (82\%) felt that the orientation prior to the implementation and use of the O-SCORE application was helpful, 10/17 (59\%) stated technical support for the application was easy to obtain, while 5/17 (29\%) had never needed any technical support.

\section{Surgical case logging}

Most residents (16/17; 94\%) felt that logging their cases using the O-SCORE application was easy. 9/17 (56\%) residents felt that the number of available cases to select from was appropriate, while two (13\%) felt there were too few cases available, and five (31\%) felt there were too many options (Figure 1). Furthermore, 10/17 (63\%) felt finding the appropriate procedure in the list was easy. Prior to the introduction of the O-SCORE application, residents used a variety of case logging systems. 8/16 (50\%) had previously used T-RES, 3/16 (19\%) used an electronic log like an excel spreadsheet, 4/16 (25\%) used a paper log and 1/16 (6\%) didn't log cases. 9/16 (56\%) of residents found logging cases on the O-SCORE system much easier as compared to T-RES, while 3/16 (18\%) found the two systems similar. Since the introduction of the O-SCORE application, residents reported logging the majority of their cases through the application. 10/16 (63\%) reported logging between $81-100 \%$ of their cases on the O-SCORE application, while 4/16 (25\%) logged between $61-80 \%$ and 2/16 (12\%) logged between 41-60\% of their cases.

\section{Assessment tool}

When a case was flagged for assessment, 9/16 (56\%) residents received in-person feedback about $25 \%$ of the time, $2 / 16$ (12\%) received in-person feedback $50 \%$ of the time, and 3/16 (18\%) received in-person feedback about $75 \%$ of the time. Timing of verbal feedback was variable; 5/16 (31\%) received the feedback in the OR immediately 
after the procedure, $5 / 16$ (31\%) received feedback after leaving the OR but on the same day, and 2/16 (12\%) received feedback the next day. Since the introduction of the OSCORE, 12/16 (75\%) of residents reported that the overall amount of verbal intraoperative feedback they receive has remained unchanged, while 4/16 (25\%) felt it has increased. 9/16 (56\%) of residents felt that staff physicians were willing to complete OSCORE evaluations when requested.

With regards to the O-SCORE evaluation form (Figure 3), residents felt that the most valuable components were the written comments section $(8 / 16 ; 50 \%)$ and the overall procedural competence field (3/16; 19\%). No residents felt the 8 Likert-scale questions were the most valuable aspect of the O-SCORE application. 12/16 (75\%) felt that the frequency at which cases were randomly selected for evaluation was appropriate. Overall, 11/16 (69\%) of residents agreed that the evaluations provided through the OSCORE were a true indicator of the level of their surgical skill. $100 \%$ of residents felt the electronic O-SCORE application made obtaining evaluations much easier compared to their previous experience with paper-based O-SCORE evaluations. Finally, 13/16 (81\%) residents felt that the O-SCORE application as a combined surgical log and evaluation tool has made an overall positive impact on their residency training.

\section{$\underline{\text { Resident comments }}$}

Residents commonly requested the ability to modify a case once it was logged so that if the actual operative procedure differed from the planned procedure (i.e simply placing a ureteric stent in the setting of a failed access ureteroscopy), this could be represented accurately in their log. They also requested a searchable feature for cases rather than having to scroll through the entire drop-down menu.

\section{Faculty responses}

Technology use in the workplace

8/12 (67\%) faculty felt comfortable with using mobile technology and 100\% felt they could easily install new applications onto their devices. 8/12 (67\%) faculty brought their iPad with them to the hospital on most or all days. 9/12 (75\%) of faculty felt that they could easily log onto the O-SCORE application, and that installing the O-SCORE application on their mobile device was easy. 7/12 (58\%) felt that technical support was easy to obtain. 9/12 (75\%) faculty had received orientation and training prior to using the application, and $100 \%$ of those who received the training felt it was helpful.

\section{Surgical case logging}

11/12 (92\%) faculty felt it was important for residents to keep a surgical log. 


\section{Assessment tool}

When cases were selected for assessment, 6/12 (50\%) faculty stated they were notified only by e-mail, while 5/12 (42\%) were additionally notified by residents either pre- or post-operatively. Faculty varied in the amount of in-person feedback they provided to a resident when they were aware that the case had been flagged for evaluation. 6/12 (50\%) reported providing verbal feedback about $50 \%$ of the cases, while $1 / 12$ (8\%) reported providing feedback $75 \%$ of the time and $3 / 12$ (25\%) reported only providing feedback $25 \%$ of the time. $2 / 12$ (17\%) never provided verbal feedback. If the faculty were to provide feedback for the flagged case, 4/12 (33\%) provided it immediately following the case and 5/12 (42\%) provided the feedback after leaving the OR but on the same day. $6 / 12$ (50\%) of faculty felt that since the introduction of the O-SCORE application, the amount of in-person feedback they provided to residents regarding their surgical performance had increased. 9/12 (75\%) faculty were willing to complete O-SCORE evaluations when requested.

Concerning the overall O-SCORE evaluation form, faculty felt that the most valuable component was the written comments section $5 / 12$ (42\%), followed by the 8 Likert scale questions $(2 / 12 ; 17 \%)$ and the independent competence indicator $(2 / 12$; 17\%). 9/12 (75\%) felt that the results of the O-SCORE evaluations were indicative of a residents overall surgical skill. 10/12 (83\%) faculty felt that the frequency of which cases were randomly selected for evaluation was adequate.

Overall, $100 \%$ of faculty felt that the electronic version made completing evaluations much easier as compared to the previously used paper format. The majority of faculty felt that the O-SCORE application as a combined surgical log and evaluation tool has had an overall positive impact on surgical training within the division (8/12; $75 \%)$.

\section{Faculty comments}

In the comments section, faculty mentioned that having to reset passwords frequently was a barrier to accessing the application. They also expressed that it was often challenging to complete evaluation forms within the 24-hour expiration time frame.

\section{Discussion}

As the paradigm of medical education shifts towards CBD, residents will need to demonstrate that they are able to competently perform entrustable professional activities (EPAs) to progress through their training and to graduate. ${ }^{2} \mathrm{CBD}$ will promote greater accountability on behalf of the resident as their training will be centered on their individual progress. With this educational model in place, surgical training programs will require more frequent, reliable and accurate assessment of a resident's technical ability in order to allow them to properly progress through their training. 
Currently in Canada, there is no nationally-accepted or well-validated surgical case log or assessment tool for use in urology residency training. The original O-SCORE assessment tool is paper-based and represents an accurate, validated tool to assess operative competency. ${ }^{5}$ The electronic application we developed streamlines the process of data collection and resident assessment by combining case-logging with O-SCORE assessment on a mobile platform.

Residents all used their iPad daily, which provided regular access to the electronic application to log their cases and review their evaluations. They felt that logging cases on the application was simple, and the vast majority of them logged all of their cases on the application. However, given their comments, some minor modifications can be made to the application to further improve its usability. Interestingly, most residents felt as though the amount of verbal feedback had not dramatically improved since the introduction of the O-SCORE application despite an obvious increase in the amount of written feedback. This likely has to do with residents still logging cases after the completion of the surgical procedure as opposed to before the case. Therefore, the staff physician may have already left the room and the opportunity for immediate verbal feedback was lost. If residents are able to modify the cases post-operatively as they requested in their comments, we may be able to increase the proportion of cases logged prior to beginning the procedure and thus increase the amount of timely verbal feedback.

The majority of faculty used their iPad regularly at the hospital, and they reported no major problems with using the O-SCORE application. Faculty felt that the written comments were the most useful portion of the O-SCORE evaluation, possibly because they allow direct identification of specific aspects of the procedure that the resident did well and/or identify areas to focus on for improvement. Overall, faculty were very willing to fill out evaluations when requested, although they did highlight that the 24-hour expiration of the evaluations was limiting. The expiration time was set so that the assessments are done shortly following the procedure in order to maximize their reliability and accuracy.

Overall, our survey results demonstrate good uptake and utilization of the electronic O-SCORE application within the urology residency training program at the University of Ottawa. Compared to previously-used paper-based forms, both faculty and residents feel the electronic platform is a more effective work-based assessment tool that facilitates both surgical case logging and assessment of technical skills. Both faculty and residents feel that the evaluations are reflective of the resident's true surgical skill and provide accurate, timely feedback to help guide improvement. These are important findings that indicate that there is willingness among both residents and faculty to utilize mobile technology during residency training and to adopt work-based assessment tools. Our study is not without its limitations. Although we feel our results demonstrate good uptake and utilization of the O-SCORE application in a single urology residency 
program, we cannot determine if the experience would be similar in other institutions or specialties. At the University of Ottawa widespread dissemination and use of iPads for clinical care has likely created a positive environment and culture for adoption of electronic applications in general. Availability of technology, as well as institutional culture and user familiarity with mobile devices likely varies across other institutions and residency programs.

Second, many of the domains we assessed regarding utilization relied on the subjective recall of survey respondents. We have not yet obtained objective data on the true frequency of use of the O-SCORE system among our staff and residents. The survey suggests the application has been received favourably, but without a direct comparison between number of cases logged/assessed and number of cases actually performed, we cannot definitely evaluate the utilization rate of the application.

In the future, as more cases are logged and assessments carried out on the application, we hope to generate learning curves for specific procedures in urology in order to determine the approximate number of cases it takes for a resident to achieve competence. Defining time to competence for core, specialty specific procedures will be critical to curriculum development in the transition to CBD for all surgical training programs.

\section{Conclusion}

Our study demonstrates that the electronic O-SCORE application is a well-accepted, user-friendly tool that may increase the frequency and quality of surgical feedback and assessments. As surgical programs begin to adopt competency-based curricula, the OSCORE mobile application can provide surgical training programs with a validated, easy to use, electronic surgical log and work-based assessment tool. 


\section{References}

1. Boerebach, B. C. M., Arah, O. A., Busch, O. R. C. \& Lombarts, K. M. J. M. H. Reliable and valid tools for measuring surgeons' teaching performance: Residents' vs. self evaluation. J. Surg. Educ. 69, 511-520 (2012).

2. Gofton, W., Dudek, N., Barton, G., and Bhanji, F. Workplace-Based Assessment Implementation Guide: Formative tips for medical teaching practice. 1st ed. (PDF) Ottawa: The Royal College of Physicians and Surgeons of Canada, pg. 1-12. (2017).

3. Fronza, J. S., Prystowsky, J. P., Darosa, D. \& Fryer, J. P. Surgical residents’ perception of competence and relevance of the clinical curriculum to future practice. J. Surg. Educ. 69, 792-797 (2012).

4. Ray, J. J. et al. Association Between American Board of Surgery In-Training Examination Scores and Resident Performance. JAMA Surg. 215, 1-6 (2015).

5. Gofton, W. T., Dudek, N. L., Wood, T. J., Balaa, F. \& Hamstra, S. J. The Ottawa Surgical Competency Operating Room Evaluation (O-SCORE): a tool to assess surgical competence. Acad. Med. 87, 1401-1407 (2012).

6. MacEwan, M. J., Dudek, N. L., Wood, T. J. \& Gofton, W. T. Continued Validation of the O-SCORE (Ottawa Surgical Competency Operating Room Evaluation): Use in the Simulated Environment. Teach. Learn. Med. 28, 72-9 (2016).

7. Doyle, J. D., Webber, E. M. \& Sidhu, R. S. A universal global rating scale for the evaluation of technical skills in the operating room. Am. J. Surg. 193, 551-555 (2007).

8. Martin, J. A. et al. Objective structured assessment of technical skill (OSATS) for surgical residents. Br. J. Surg. 84, 273-278 (1997). 


\section{Figures and Tables}

Fig. 1. O-SCORE application - faculty selection.

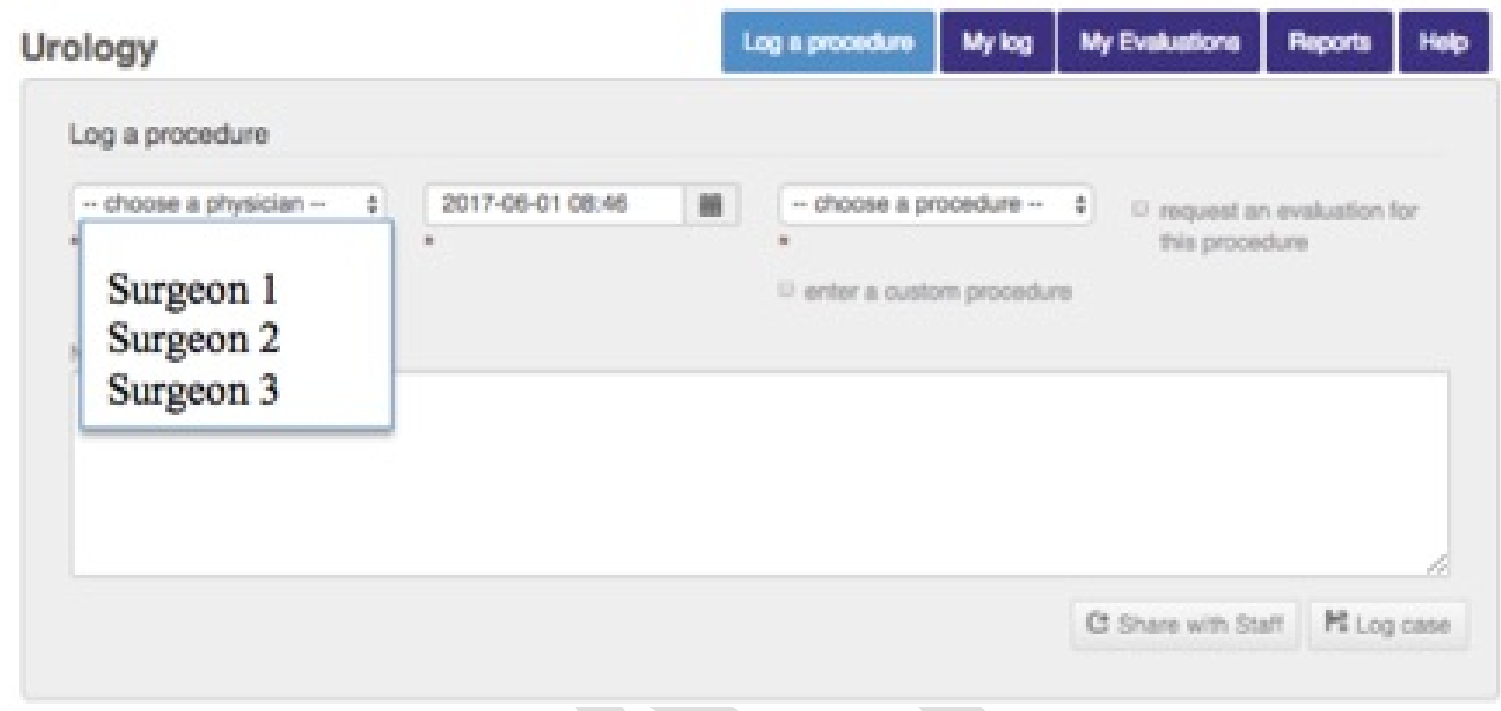

Fig. 2. O-SCORE application - case selection.

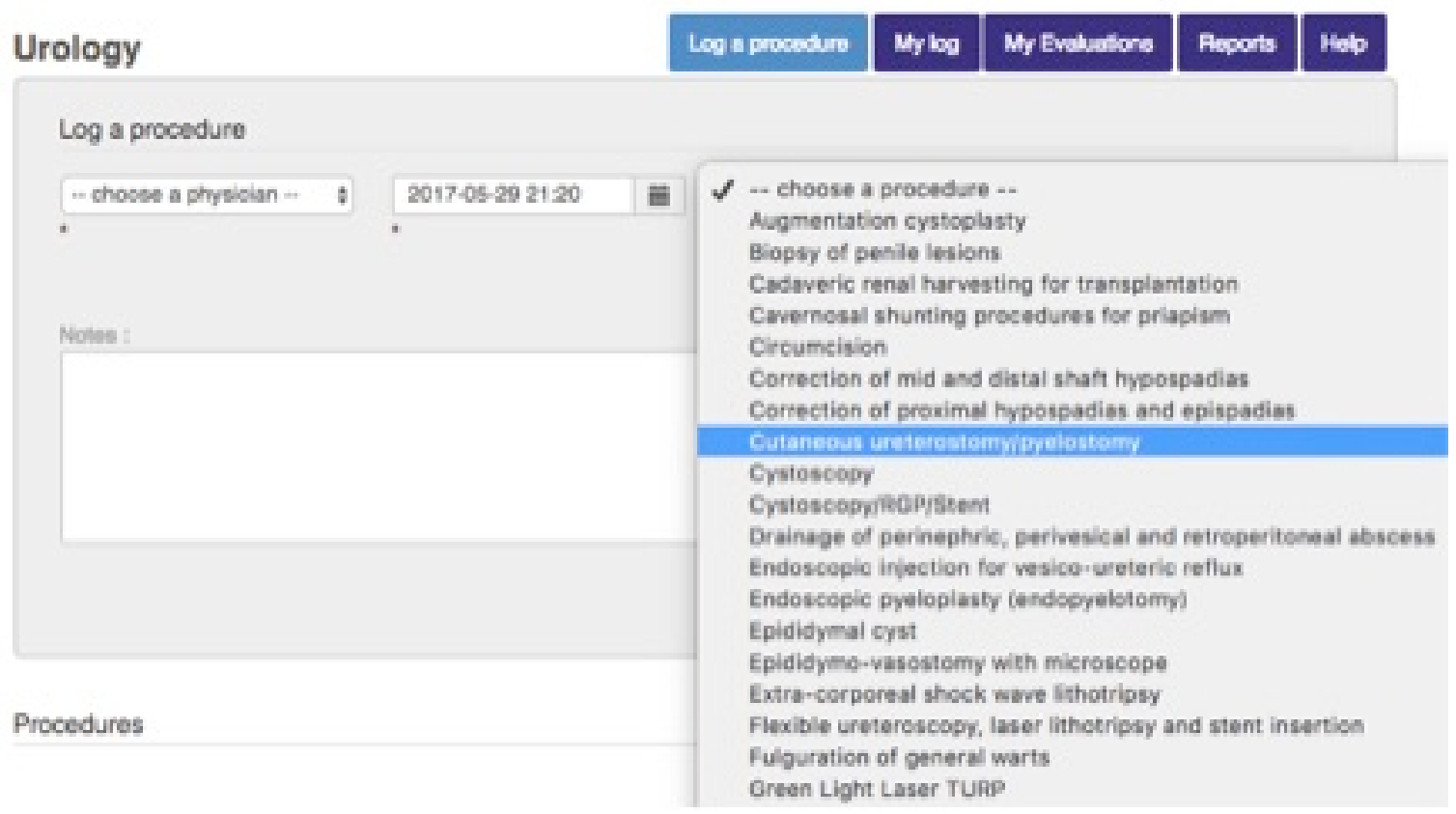


Fig. 3. O-SCORE application - evaluation form.

1. Pre-procedural Plan

Gathers/assesses requ

2. Case Preparation complications

3. Knowledge of Specific Procedural Steps

1

3

3

4

5

Understands steps of procedure, potential risks and means to avoid / overcome them

4. Technical Performance

1

2

3

4

5

Efficiently performs steps avoiding pitfalls and respecting soft tissues

5. Visuospatial Skills

1

2

3

4

5

3D spatial orientation and able to position instruments / hardware where intended

6. Post procedural plan

1

2

3

4

4

5

Obvious planned course of procedure with economy of movement and flow

8. Communication

1

2

3

$(4)$

5

Professional and effective communication / utilization of staff

9. Resident is able to safely perform this procedure independently

Yes No

10. Give at least 1 specific aspect of procedure done well

Overall very well done. good knowledge of steps. good use of hook and harmonic. good safe application of stapler on vein. 
Fig. 4. Graphical representation of key survey results.
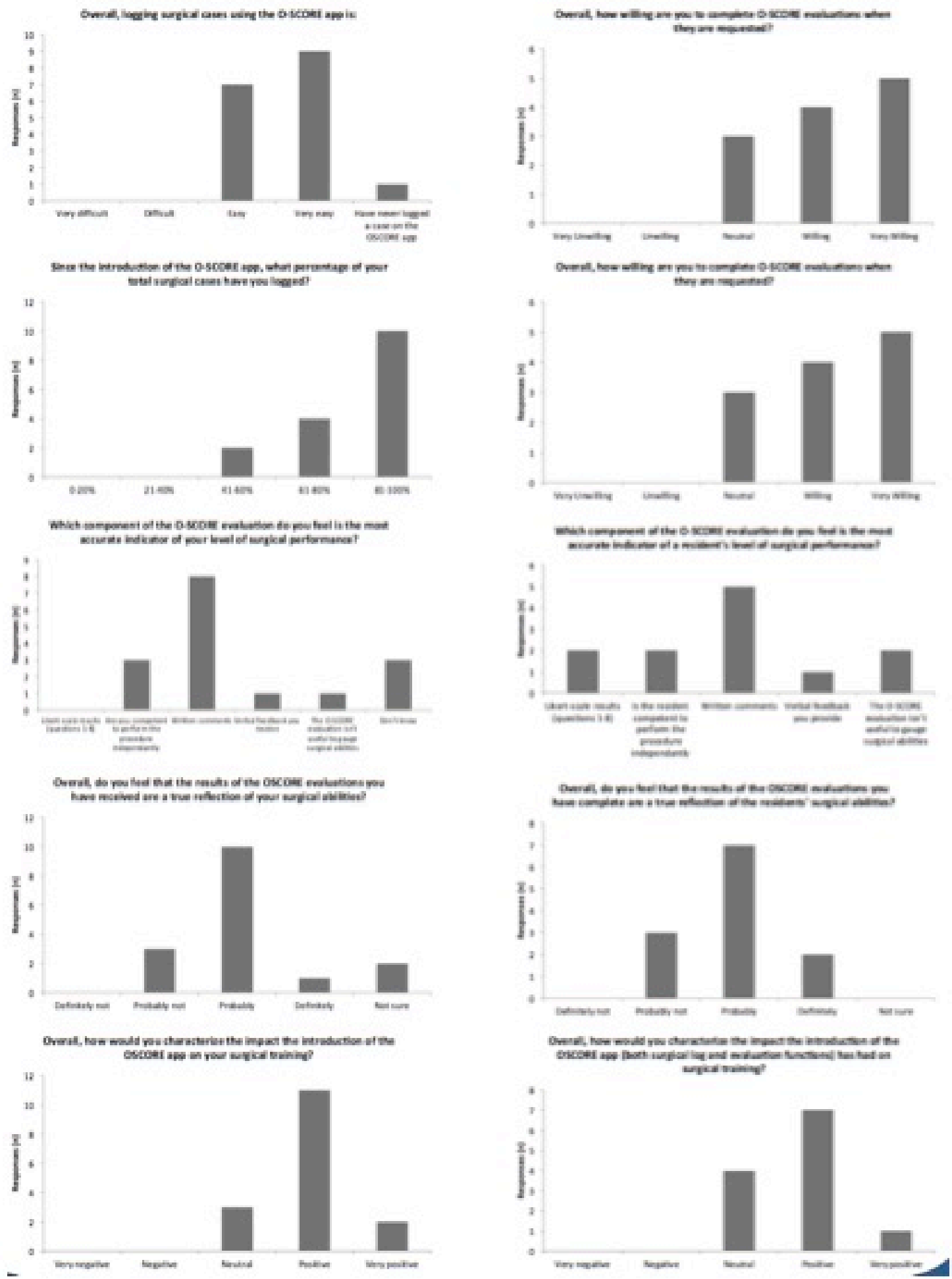